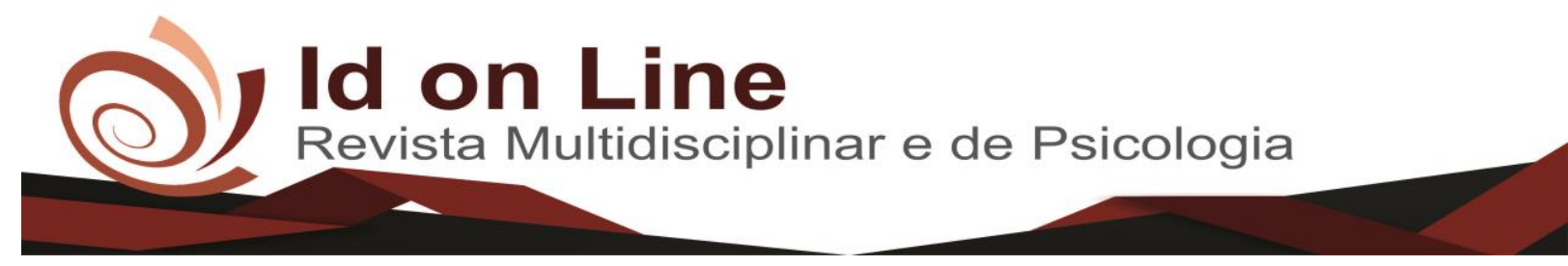

Artigo de Revisão

\title{
Sintomatologia dolorosa após tratamento endodôntico: Revisão da literatura
}

\author{
Roberta Karien Ferraz Oliveira ${ }^{1}$; Marcelo Pereira da Rocha ${ }^{2}$
}

\begin{abstract}
Resumo: Entre sessões ou até mesmo após a finalização do tratamento endodôntico, a dor pode estar presente por uma inflamação aguda dos tecidos perirradiculares ou em decorrência de alguma agressão ao canal radicular. $\mathrm{O}$ tratamento endodôntico é uma das terapias odontológicas mais associadas à sintomatologia dolorosa pós operatória, se comparada a outras áreas da Odontologia. Objetivo: Revisar a literatura sobre sensibilidade dolorosa após tratamento endodôntico. Metodologia: Revisão sistemática da literatura, do período 2010 a 2018. Resultados esperados: Espera-se realizar uma síntese do conhecimento acerca da sensibilidade dolorosa após tratamento endodôntico, com vistas a conhecer os fatores associados e formas de prevenção.
\end{abstract}

Palavras-chave: Dor Pós-Operatória; Endodontia; Canal Radicular.

\section{Pain symptomatology after endodontic treatment: Literature review}

\begin{abstract}
Between the moments of a termination of the endodontic treatment, a pain may be present in an acute inflammation of the periradicular tissues, in relation to some aggression to the root canal. Endodontic treatment is one of the most common dental therapies to the painful symptomatology of surgery, compared to other areas of dentistry. Objectives: To analyze the evolution of the painful disease after the endodontic treatment and correlated with the factors of age, sex, tooth treatment, pulmonary diagnosis, intracanal medication used and number of sessions. Methodology: we intend to obtain an analysis of data from medical records of patients submitted to endodontic treatment in the last six years between June 2012 and June 2018. Results obtained: low number of patients with painful disease after endodontic treatment The postgraduate measures in Endodontics of Vitória da Conquista, State of Bahia, with higher frequency for the previous symptomatology and the correct ones during the operative act.
\end{abstract}

Keywords: Postoperative Pain; Endodontics;Root Canal.

\section{Introdução}

A perfeita desinfecção, preparação químico-mecânica e obturação do sistema de canais radiculares são funções da Endodontia (CARVALHO, 2017). Dentes submetidos à terapia endodôntica tem uma taxa de sucesso elevada.

\footnotetext{
${ }^{1}$ Graduanda em Odontologia, pela Faculdade Independente do Nordeste - FAINOR, Vitória da Conquista, Bahia, Brasil. Contato: robertinha_ferraz@hotmail.com;

${ }^{2}$ Mestre em Saúde Coletiva, Docente do curso de Odontologia da Faculdade Independente do Nordeste - FAINOR, Vitória da Conquista, Bahia, Brasil.
}

696 Id on Line Rev. Mult. Psic. V.12, N. 42, p. 696-703, 2018 - ISSN 1981-1179

Edição eletrônica em http://idonline.emnuvens.com.br/id 
Porém, apesar de grandes avanços na área, nos últimos anos, a sintomatologia dolorosa se apresenta em parte dos pacientes (WATANABE, 2012) com uma taxa maior se comparado a outros procedimentos odontológicos (PALMA, CENTENARO, SILVA e MARTINAZZO, 2017).

A dor orofacial implica em danos físicos e psicológicos ao paciente (KUROIWA et al, $2011)^{4}$ e, quanto maior a agressão tecidual, mais elevada será a intensidade da dor, especialmente no período pós-operatório, o que acarreta ansiedade e desconforto ao indivíduo, repercutindo negativamente no decorrer do tratamento (FERREIRA, TRAVASSOS, ALBUQUERQUE e OLIVEIRA, 2017).

Entre sessões ou até mesmo após a finalização do tratamento endodôntico, a dor pode estar presente por uma inflamação aguda dos tecidos perirradiculares, em decorrência de alguma agressão ao canal radicular (CARVALHO, 2017). Além de induzida, pode ser exacerbada a depender do estado de saúde do paciente, da sua condição bucal, dos sintomas clínicos, do dente em questão e do procedimento realizado (FONTENELE, et al, 2016).

A sensibilidade dolorosa pode ocorrer por razões microbianas, mecânicas ou químicas (CARVALHO, 2017). A extrusão apical de detritos infectados durante a instrumentação é o principal fator etiológico da inflamação periapical e da dor pós-operatória na maioria dos casos (SAHI, ASGHARI, RAHIMI et al, 2016). Aparece em pacientes com histórico de dor antes do tratamento endodôntico, muito embora existam aqueles anteriormente assintomáticos (FERREIRA, TRAVASSOS, ALBUQUERQUE e OLIVEIRA, 2017).

Dentes previamente tratados endodonticamente e com endodontia iniciada são mais propensos à dor. Além disso, a idade é um fator de risco, especialmente a partir de 50 anos de idade. Um tratamento endodôntico bem realizado, com total assepsia, evita o pós operatório de sintomatologia dolorosa (NAIR, RAHUL, DEVADATHAN e MATHEW, 2017). Apesar de todas as técnicas de instrumentação resultarem em extrusão apical, há estudos que mostram que algumas técnicas rotativas minimizam essa ocorrência (SHAHI, ASGHARI e, RAHIMI, 2016).

Várias estratégias foram investigadas para alívio da dor pós tratamento endodôntico. Entre eles, existem métodos farmacológicos, com uso de analgésicos, corticosteroides, antiinflamatórios não esteroidais (NSAIDs), anestésicos e antibióticos, além de redução oclusal e hipnose (IRANMANESH, PARIROKH, HAGHDOOST e ABBOTT, 2017). 
O conhecimento da ocorrência da dor pós-operatória associada ao tratamento endodôntico, especialmente sobre sua causa e recorrência, é de grande valia para que o Cirurgião-Dentista efetive ações que visem a prevenção e o desenvolvimento de um melhor tratamento (JAYAKODI, 2012), aliando o correto diagnóstico ao tratamento efetivo com correta utilização medicamentosa (KUROIWA , 2011).

Diante disso, este estudo objetivou revisar a literatura acerca da ocorrência de sensibilidade dolorosa após a realização de tratamento endodôntico.

\section{Material e Métodos}

Esta pesquisa foi conduzida por meio de revisão de literatura, buscando-se uma síntese do conhecimento da temática em questão, partindo das seguintes perguntas de pesquisa: quais são os principais fatores de risco e formas de prevenção da sensibilidade dolorosa pós tratamento endodôntico? O que apontam os estudos referente à incidência da dor pós-operatória em endodontia?

Para tanto, foram utilizadas as seguintes palavras-chave: dor, pós-operatório e endodontia, e suas respectivas em inglês pain, postoperative endodontics, para acesso às Bases de dados: ScientificElectronic Library (SciELO), PUBMED, Literatura Latino-Americana e do Caribe em Ciências da Saúde (LILACS), e BIREME.

O período pesquisado compreendeu de 2010 a 2018, com intuito de obter acesso aos trabalhos mais recentes sobre o assunto em questão.

Foram incluídos somente artigos científicos, dentro da temática proposta, selecionado por meio da leitura do título e do resumo, com textos completos, publicados nas línguas portuguesa e inglesa. Por sua vez, foram adotados os seguintes critérios de exclusão: trabalhos em outro formato que não artigo, como teses e dissertações; os que fogem da temática proposta, artigos incompletos e publicados em outros idiomas que não os citados anteriormente. 


\section{Resultados e discussão}

Estudo conduzido por meio de revisão da literatura ressaltou a importância de se conhecer as causas e mecanismos relacionados à dor entre as sessões na endodontia, de modo a propiciar ao clínico as condições para prevenção e controle adequado dessa condição indesejável. Os fatores causais da dor incluem lesões mecânicas, químicas e microbianas na polpa ou nos tecidos perirradiculares, induzidos ou exacerbados durante o tratamento do canal radicular. Ainda sublinha diversas modalidades de tratamento para o alívio da dor e edema em tais situações, incluindo uso de medicação prévia à intervenção, drenagem, alívio da oclusão e medicação intracanal e sistêmica (JAYAKODI, 2012).

Pesquisa realizada entre 2015 e 2017 avaliou a frequência e os fatores relacionados à dor pós-tratamento endodôntico, como idade, diagnóstico inicial, quantidade de canais radiculares, tipo de dente, gênero sexual, tipo de solução irrigadora utilizada e o número de sessões para a conclusão da endodontia. Do total de 454 dentes que receberam o tratamento apenas 3,5\% apresentou flare-up. Necrose pulpar sem lesão periapical representou 5,9\% dos casos, seguido por por necrose pulpar com lesão periapical $(5,3 \%)$ e pulpite irreversível $(2,2 \%)$. Também foi observado que pacientes submetidos a um número maior de sessões desenvolveram com maior frequência sintomatologia dolorosa, quando comparados aos casos realizados em sessão única. Em relação à idade, tipo de dente, sexo, número de canais e a solução irrigadora utilizada não houve diferença significativa (FARZANA et al, 2010). A incidência de flare-up foi insignificante nos tratamentos realizados em sessão única. Lesão periapical em dentes com necrose foi fator significativo para desenvolvimento de dor pós operatória (ALSHERI, 2018).

Estudo prospectivo, realizado com quarenta e seis voluntários, buscou avaliar a dor pós-operatória após terapia endodôntica, com e sem alargamento foraminal, em dentes unirradiculares com polpa necrótica. Os pacientes foram divididos aleatoriamente em dois grupos distintos, de acordo com os comprimentos de trabalho estabelecidos. Um grupo que recebeu a denominação de FE teve o seu comprimento de trabalho estabelecido no limite apical, enquanto no grupo controle a instrumentação foi realizada 1,0 $\mathrm{mm}$ aquém do ápice, ambos grupos tiveram seus canais instrumentados com o instrumento rotatório Reciproc R40 (VDW, Munique, Alemanha). Os participantes registrarem sua dor em quatro categorias, nenhuma, 
leve, moderada ou grave, com uso de escala nas primeiras 24 horas, 72 horas e após 1 semana de realização do tratamento. Do toral, $17,8 \%$ dos pacientes relataram algum tipo de dor. Houve maior proporção de pacientes do grupo FE que relataram a presença de uma dor leve nas primeiras 24 horas, quando comparados com o grupo controle. Quanto ao prazo de 72 horas e 1 semana não houve diferenças significativas entre os grupos (CRUZ JUNIOR et al, 2016).

Estrela e colaboradores (ESTRELA, 2016), em uma revisão sistemática da literatura avaliou se a entrada ou continuação da proliferação de bactérias nos sistemas de canais radiculares dentário eram as principais responsáveis pelo insucesso do tratamento endodôntico e pela sintomatologia dolorosa pós tratamento, onde puderam constatar que a alta incidência de Enterococcus faecalis nos condutos radiculares produziam uma quantidade exacerbada de toxinas, provocando assim a sintomatologia dolorosa e que a não regressão da mesma levava à necessidade de retratamento destes dentes.

Filho EDG et al (2010) avaliaram a dor pós-operatória em dentes vitais submetidos ou não ao alargamento foraminal até a lima \# 30. Quarenta dentes foram selecionados e divididos randomicamente em dois grupos. No Grupo I (experimental) houve ampliação foraminal até a lima \# 30 e comprimento de trabalho 1 a $2 \mathrm{~mm}$ aquém do forame. No Grupo II (controle) utilizaram limite de trabalho de 1 a $2 \mathrm{~mm}$ aquém do ápice e manutenção do remanescente pulpar. Todos as endodontias foram realizadas em sessão única, com uso de localizador apical para a determinação do comprimento de trabalho, além de radiografia de comprimento de trabalho. A dor foi avaliada nos períodos de 24 e 48 h. Não foi observada diferença estatística entre os dois grupos. O alargamento do forame apical não aumentou a incidência de dor, de acordo com os relatos dos pacientes.

Estudos realizados por Farzana e colaboradores (2010), buscaram avaliar a incidência de dor pós-operatória após a realização de tratamento endodôntico em 52 dentes com polpa vital ou necrosada. Foi observado que a dor leve ocorreu em 4,34\% dos pacientes que apresentavam a polpa em estado vital, e 4,39\% em dentes não vitais, onde ele pôde concluir que não havia uma diferença considerável na ocorrência da dor entre dentes com polpas vitais e não vitais. Confirmando assim o estudo de Palma e Centenaro e Martinazzo (2017) que relataram evidências onde pacientes diagnosticados com pulpite irreversível não desenvolveram dor pós-operatória quando o adequado preparo mecânico-químico do conduto radicular foi realizado. 
Estudo realizado por Raldi, Oliveira e Lage-Marques (2012), observaram a dor póstratamento endodôntico quando os pacientes faziam uso previamente de dexametasona oral. $\mathrm{Na}$ primeira sessão os canais eram instrumentados, colocava uma bolinha de algodão estéril e selados com Cavit. Aleatoriamente os pacientes recebiam uma dosagem de $0,75 \mathrm{mg}$ de dexametasona ou placebo, e então era feito um contato com os pacientes 8 e 24 horas após a realização do atendimento. O grupo de pacientes que receberam a dexametasona relataram menos dor quando comparados com o placebo. Entretanto o uso de anti-inflamatório esteroidal na rotina clínica deve ser questionado, uma vez que deve ser levado em conta os riscos que o mesmo apresenta ao organismo necessitando dessa forma de um estudo mais aprofundado acerca do assunto.

Também foram observadas controvérsias em relação à técnica de instrumentação, escolha da substância de irrigação, da medicação intracanal (FARZANA et al, 2010) e da quantidade de sessões realizadas, ressaltando a importância de estudos futuros para análise da influência desses fatores no desencadeamento da sintomatologia dolorosa pós-tratamento endodôntico (SHAHI S, ASGHARI V, RAHIMI, 2016; JAYAKODI, 2012; CRUZ JUNIOR et al, 2016; FARZANA, 2010).

\section{Conclusão}

Apesar do índice de flare-up, não influenciar de forma significativa sobre o resultado da terapia endodôntica (NAIR, RAHUL, DEVADATHAN e MATHEW, 2017), a sua ocorrência é indesejável tanto por parte do profissional quanto do paciente, podendo influenciar de maneira direta na relação destes (FERREIRA, TRAVASSOS, ALBUQUERQUE e OLIVEIRA, 2017). Sendo assim o Cirurgião-Dentista deve empregar medidas adequadas a fim de impedir o desenvolvimento desses episódios dolorosos após a realização dos tratamentos endodônticos.

Embora a existência de limitações no presente estudo, a literatura pesquisada possibilitou uma síntese do conhecimento referentes aos episódios de dor após a realização de tratamento endodôntico. Os trabalhos apresentaram alguns aspectos em comum como o fato do paciente que apresenta dor pré-operatório é susceptível ao desenvolvimento dessa após a 
realização da terapia, e que o uso de medicamentos previamente pode diminuir a incidência destes eventos dolorosos. Houveram discordâncias quanto às questões de estado da vitalidade pulpar e a técnica de alargamento foraminal.

Recomenda-se então a realização de novos estudos de modo que se possa conhecer melhor acerca dos fatores desencadeadores da sintomatologia dolorosa pós-terapia endodôntica.

\section{Referências}

ALSHERI AA, et al., Endodontic Flare-Ups: A Study of Incidence and Related Factors. The Egyptian Journal of Hospital Medicine. 2018; 70(2): 349-53

BORLINA SC, DE SOUZA V, HOLLAND R, MURATA SS, GOMES-FILHO JE, DEZAN JUNIOR E, et al.Influence of apical foramen widening and sealer on the healing of chronic periapical lesions induced in dogs' teeth Oral Surg Oral Med Oral Pathol Oral Radiol Endod. $2010 ; 109(6): 932-40$.

CARVALHO CF. Flare-up em Endodontia: principais fatores etiológicos. Porto. Dissertação [Mestrado em Medicina Dentária] - Universidade Fernando Pessoa; 2017.

CRUZ JUNIOR JA, COELHO MS, Kato AS, VIVACQUA-GOMES N, FONTANA CE, ROCHA DG, et al. The effect of foraminal enlargement of necrotic teeth with the reciproc system on postoperative pain: a prospective and randomized clinical trial. $J$ Endod. 2016;42(1):8-11.

ESTRELA C, et al. Dor pós-operatória em dentes com inflamação pulpar - revisão sitemática. Rev Odontol do Brasil Central 2016; 15(40): 1-12

FARZANA M. F. et al. Postoperative pain following multi-visit root canal treatment of teeth with vital and non-vital pulps. Journal of Armed Forces Medical College, Bangladesh. 2010; 6(2): 6-15.

FERREIRA GS, TRAVASSOS RMC, ALBUQUERQUE DS, OLIVEIRA IKC. Incidência de flare-ups na clínica de endodontia da FOP-UPE. Revista de Odontologia da Universidade Cidade de São Paulo. 2017; 19(1):33-8.

FILHO EDG, et al. Avaliação in vivo da dor pós-operatória em dentes vitais após o alargamento do forame apical. Rev Fed Odontol. 2010; 15(2): 145-9.

FONTENELE JWN, CASTRO IPD, PEDROSA MS, FONTENELE MNB, POMPEU JGF, 
DELBONI MG. Incidence and factors related to endodontic flare-ups: a literature review. Salusvita. 2016; 35(4):547-61.

IRANMANESH F, PARIROKH M, HAGHDOOST AA, ABBOTT PV. Effect of Corticosteroids on Pain Relief Following Root Canal Treatment: A Systematic Review. Iranian Endodontic Journal. 2017; 12(2):123-30. doi:10.22037/iej.2017.26.

JAYAKODI $\mathrm{H}$, et al. Clinical and pharmacological management of endodontic flare-up. $J$ Pharm Bioallied Sci, Delhi. 2012;4 (2):294-8.

JAYAKODI H, KAILASAM S, KUMARAVADIVEL K, THANGAVELU B, MATHEW S. Manejo clínico e farmacológico do surto endodôntico. Journal of Pharmacy \& Bioallied Sciences . 2012; 4(2):11-5. doi: 10.4103 / 0975-7406.100277.

KUROIWA DN, MARINELLI JG, RAMPANI MS, OLIVEIRA W, NICODEMO D. Desordens temporomandibulares e dor orofacial: estudo da qualidade de vida medida pelo Medical Outcomes Study 36 - Item Short Form Health Survey. Revist da dor. 2011; 12(2):938. http://dx.doi.org/10.1590/S1806-00132011000200003

NAIR M, RAHUL J, DEVADATHAN A, MATHEW J. Incidence of Endodontic Flare-ups and Its Related Factors: A Retrospective Study. Journal of International Society of Preventive \& Community Dentistry. 2017; 7(4):175-9. doi:10.4103/jispcd.JISPCD_61_17.

PALMA LZ, CENTENARO WL, SILVA CA, MARTINAZZO CA. Incidence of pain after endodontic treatment conclusion in permanent teeth in patients treated in clinic of dentistry school of URI Erechim. Perspectiva. 2017; 41(153):73-83.

RALDI, D. P.; OLIVEIRA, R. B.; LAGE-MARQUES, J. L. Medicação sistêmica como coadjuvante do tratamento endodôntico. APCD Revista, v. 56, n. 5, set/ out. 2012

SHAHI S, ASGHARI V, RAHIMI S, et al. Postoperative Pain after Endodontic Treatment of Asymptomatic Teeth Using Rotary Instruments: A Randomized Clinical Trial. Iranian Endodontic Journal. 2016; 11(1): 38-43. doi:10.7508/iej.2016.01.008.

WATANABE VM. Índices de sucesso do retratamento endodôntico: uma revisão de literatura. Piracicaba. Monografia [Especialização em Endodontia] - Faculdade de Odontologia de Piracicaba da Universidade de Campinas; 2012.

\section{Como citar este artigo (Formato ABNT):}

OLIVEIRA, Roberta Karien Ferraz; ROCHA, Marcelo Pereira da. Sintomatologia dolorosa após tratamento endodôntico: Revisão da literatura. Id on Line Rev.Mult. Psic., 2018, vol.12, n.42, p. 696703. ISSN: 1981-1179.

Recebido: 15/10/2018;

Aceito: 17/10/2018 